\section{El Programa de Extensión de \\ Economía Social y Solidaria de la Universidad Nacional del Litoral}

Julio C. Tealdo

Docente investigador de la Universidad

Nacional del Litoral, Argentina.

Director del Programa de Extensión

Economía Social y Solidaria.
Economía Social y Solidaria /

Desafíos de gestión

RECEPCIÓN: 26/06/15

ACEPTACIÓN FINAL: 25/08/15

\section{Resumen}

La Economía Social y Solidaria (ESS) en el ámbito universitario argentino ha alcanzado una institucionalización en las diferentes universidades de Argentina. La Universidad Nacional del Litoral no es ajena a esta situación, y en el año 2013 se crea el Programa de Extensión de Universidad Nacional y Solidaria. De esta manera, el presente artículo desarrolla los objetivos, líneas de acción y las principales acciones realizadas en la extensión, docencia e investigación, del Programa. Se concluye con una reflexión del camino recorrido destacando los desafíos de gestión, limitaciones y logros en el campo de la ESS.

Palabras clave

- Programa de extensión

- Economía Social y Solidaria

- gestión

- Universidad Nacional del Litoral

\section{Resumo}

A economia Social e Solidária (ESS) no campo universitário argentino há atingido uma institucionalização nas diferentes universidades da Argentina. A Universidade Nacional do Litoral não é estranha a esta situação e no ano 2013 cria-se o Programa de Extensão da Universidade Nacional e Solidária. O presente artigo desenvolve os objetivos, linhas de ação e as principais ações realizadas na extensão, ensino e pesquisa do Programa. Conclui-se com uma reflexão do caminho percorrido destacando os desafios de gestão, limitações e conquistas no campo da ESeS.

Palavras-chave

- Programa de extensão

- Economia Social e Solidária

- gestão

- Universidade Nacional do Litoral

Para citación de este artículo

Tealdo, J. C. (2015). El Programa de Extensión de Economía

Social y Solidaria de la Universidad Nacional del Litoral.

En Revista +E versión digital, (5), pp. 136-141. Santa Fe,

Argentina: Ediciones UNL. 


\section{C. \\ las distintas estrategias vinculan el ámbito académico -incluyendo a universidades latinoamericanas - con distintos actores (gubernamentales, económicos y sociales) de la región santafesina.}

\section{La Economía Social y Solidaria (ESS),} breves consideraciones conceptuales

El sistema capitalista a través del tiempo se ha convertido en un sistema económico altamente dinámico que ha generado grandes cambios no sólo económicos sino también sociales, políticos, culturales, institucionales, territoriales, etc. (Bowles y Edwars, 1985). Desde una perspectiva histórica, este sistema no tiene más que unos 600 años de vigencia, y ha pasado por distintas etapas y se ha ido consolidando en cada vez más países y regiones del mundo como un sistema hegemónico, a pesar de no ser el único que existe en la actualidad. Las crisis de la economía-mundo Rapoport y Brenta (2010) iniciada hacia los años 70 del siglo pasado y la evidente reestructuración del sistema capitalista mundial han puesto en debate al propio sistema económico imperante en la gran mayoría de los países como nunca antes. Este debate ha impactado en las ciencias económicas, donde empieza a aparecer la llamada otra economía. Analizando el marco teórico de lo que entendemos por otra economía, encontramos distintos conceptos de los cuales no todos son coincidentes pero sí tienen puntos en común. Tomaremos en consideración algunos de ellos y esbozaremos una conceptualización de lo que entendemos como ESS desde el Programa de Extensión de Economía Social y Solidaria de la UNL. Distintos autores, sobre todo en América Latina, han venido haciendo un recorrido sobre lo que comprende esta nueva conceptualización. Tenemos, por un lado, aquellos que hablan de economía solidaria, como Singer (2000), Da Cruz (2006); de economía de solidaridad, como Razeto (1997); de economía popular, como Coraggio (2005). También los enfoques que piensan a la economía social como surgimiento exclusivamente de una crisis friccional o coyuntural (Arroyo, 2008 y Noseto, 2005), o el enfoque que considera a la ESS (Coraggio, 2005; 2007) basada en las relaciones sociales de intercambio (Polanyi, 2011). Desde la UNL y en el enfoque del Programa consideramos que la ESS se refiere a una concepción del mundo y de la vida alternativa a la lógica dominante (el sistema capitalista). Es un conjunto de prácticas fundadas en relaciones de colaboración y solidaridad, inspiradas por valores que ubican al ser humano como sujeto $y$ finalidad de la actividad económica, desestimando la acumulación privada de riqueza y de capital (Lafferriere; Tealdo; Azerrad, 2010). Esta concepción de la economía se constituye en un proyecto para la sociedad, como una forma de inclusión social y de promoción de una cultura solidaria. El fin es apostar a un mejoramiento para la comunidad en general, garantizar la propia vida y la de la comunidad sustentados en:

- la redistribución (no en la acumulación);

- la obtención de los recursos necesarios para mantener el capital de trabajo, la reproducción de la vida y la unidad doméstica (no en la máxima ganancia),

- la reproducción de los valores de solidaridad, cooperación, asociatividad, reciprocidad (no en la estrategia de economía de escala como herramienta de acumulación y maximización de la ganancia).

En síntesis, en un sistema económico generador de sociedad y no de utilidad (Lafferriere; Tealdo; Azerrad, 2010). 


\section{El Programa de Extensión Economía Social y Solidaria (PEESS) en la UNL: antecedentes, objetivos y líneas de acción} Desde el año 2006, en la UNL se desarrollan acciones de diversa naturaleza vinculadas al debate de la ESS. Podemos citar como referencia las primeras actividades en torno a la temática: la charla debate de la empresa recuperada Zanon en el año 2006 y la concreción de los Coloquios de Economía Social y Solidaria, desarrollo local y organizaciones de la sociedad civil realizados en los años 2007 y 2010.

Luego de una serie de actividades y proyectos atinentes a la ESS (consultar Tealdo, 2014), en el año 2013 se aprueba el PEESS en el marco de la Secretaría de Extensión de la UNL, con sede en la Facultad de Ciencias Económicas (Resolución CS № 480/13). De esta manera, la temática de la ESS se institucionaliza y define objetivos y líneas estratégicas de trabajo. El Programa posee un director y un Consejo de Dirección que está integrado por representantes de las diferentes unidades académicas de la Universidad.

Entre los objetivos del Programa figuran:

- Propiciar la creación, desarrollo y fomento de acciones sustantivas de investigación, docencia y extensión en el marco de la ESS, tendientes a consolidar y potenciar, desde una perspectiva multi e interdisciplinaria, a los actores y las prácticas que la constituyen.

- Aportar, desde un enfoque interdisciplinario, a la reflexión crítica y análisis respecto del rol de las políticas públicas en el fortalecimiento de la ESS, que a su vez promueva el desarrollo local en la región. - Promover la consolidación de valores que construyen lazos sociales conjugando iniciativa y solidaridad como un modo de aportar a la sostenibilidad.

Y entre los objetivos específicos aparecen:

- Impulsar la articulación del área de extensión con la investigación, que aporten una perspectiva teórica, epistemológica y metodológica en el marco de ESS.

- Apoyar las mejoras de la calidad e innovación de los sistemas, instituciones y prácticas de educación y formación profesional en el marco de la ESS.

- Fortalecer las prácticas socioproductivas de los actores de la ESS de la región, propiciar la adquisición y uso de conocimientos, habilidades y destrezas que permitan el desarrollo personal y productivo.

- Establecer, articular, fomentar y fortalecer relaciones y mecanismos de acompañamiento a las instituciones de apoyo y fomento de la ESS que procuran incidir en las decisiones de los actores políticos y sociales en el diseño de políticas públicas. - Desarrollar mecanismos institucionales que permitan relacionar las agendas de las políticas públicas con las problemáticas de las iniciativas socioproductivas y las líneas de intervención de la Universidad, a fin de potenciar las acciones públicas de fortalecimiento de la ESS.

\subsection{Las líneas de acción y estrategias de abordaje}

EI PESS involucra líneas de trabajo que son abordadas desde la investigación, la docencia y la extensión. Las distintas estrategias vinculan el ámbito académico -incluyendo a universidades latinoamericanas - con distintos actores (gubernamentales, económicos y sociales) de la región santafesina.

Entre las líneas prioritarias, merecen destacarse:

- ESS y educación. La educación en ESS, las acciones concretas en diferentes niveles educativos, la educación popular.

- ESS y culturas. Nuevas formas de relaciones entre economía y diversidad cultural. Experiencias autogestivas artísticas y revalorización de tradiciones.

- ESS y mercados solidarios. Nuevas formas de comercialización: ferias francas, ferias artesanales, trueque, cadenas solidarias, mercados solidarios y comercio justo. Análisis y estudio de las nuevas formas de construcción de mercados alternativos y solidarios en la producción de bienes y servicios (diseño de productos, estrategias y nuevas formas de comercialización, costos, gestión para el desarrollo de la ESS).

- ESS, mutualismo y cooperativas. Primera generación de mutuales y cooperativas rurales y urbanas: promoción y revitalización.

Segunda generación de cooperativas y mutuales (emergentes de la Argentina poscrisis 2001): cooperativas de hábitat e infraestructura urbana; de bienes y servicios; de reciclajes y redes de promotores ambientales (cartoneros).

- ESS y género. Visibilización de la mujer en las actividades de ESS. Mujer y trabajo doméstico y reproducción ampliada de la vida. Diferentes experiencias del trabajo cooperativo femenino (en ámbitos rurales y urbanos).

- ESS y la cuestión ambiental. Valorización de la naturaleza en la conformación de emprendimientos de ESS. Turismo: creación de espacios turísticos con presencia de mercados solidarios, responsables y sustentables.

- ESS y nuevas ruralidades. Sistemas alimentarios autogestionados, formas alternativas de producción familiar y de pequeños y medianos productores. Soberanía alimentaria: promoción de las huertas comunitarias. Redes de emprendimientos de producción de alimentos que nuclean asociaciones de comercio justo, consumo responsable y organizaciones no gubernamentales. Nuevos movimientos sociales de defensa de la tierra, agricultura ecológica y generación de formas de laboreo en consonancia con la ESS. - ESS, asociaciones barriales, vecinales y ONG. La participación ciudadana en la promoción de la ESS. Los espacios de las vecinales como impulsoras del trabajo asociativo y la conformación de cooperativas de trabajo vinculadas a la construcción, la producción textil y el cuidado de personas, entre otras actividades. - ESS y software libre. El trabajo en red de programadores: principales acciones y difusión de este tipo de software. Fomento y uso de software libre en instituciones públicas y emprendimientos vinculados a la economía social. 


\author{
a través de distintas modalidades, \\ el PEESS apoya, coordina, fomenta \\ y articula diferentes proyectos \\ de extensión; líneas de capacitación \\ y espacios de trabajo asociativos \\ que potencian y promuevan políticas \\ públicas pertinentes para este tipo \\ de economía
}

\begin{abstract}
- Políticas públicas y ESS. Articulación de diferentes escalas estatales, organizaciones de la sociedad civil y beneficiarios para el desarrollo de las políticas públicas (sociales y de empleo). Las políticas de financiamiento, la promoción de las cooperativas de trabajo y de empresas recuperadas.

- Finanzas solidarias y microcréditos. Instrumentos, instituciones y nuevas formas de finanzas solidarias en Argentina y Latinoamérica. - ESS y Desarrollo. Debates de la ESS en el Norte y el Sur. Los estudios decoloniales y la antropología del desarrollo.

- ESS y comunicación. Radios comunitarias y escolares.

Cooperativas radiales y audiovisuales. Cooperativas de imprenta, arte y diseño. Nuevas políticas comunicacionales y ESS.

Si bien, el PEESS no se encuentra desarrollando todas las líneas de acción, al interior de la UNL los diferentes equipos de trabajo se enmarcan en algunas de ellas vinculadas a la extensión, docencia e investigación.
\end{abstract}

\section{Las acciones de extensión del PEESS}

A través de distintas modalidades, el PEESS apoya, coordina, fomenta y articula diferentes proyectos de extensión; líneas de capacitación y espacios de trabajo asociativos que potencian y promuevan políticas públicas pertinentes para este tipo de economía.

\subsection{Proyectos de extensión de la UNL}

En lo que respecta a proyectos de extensión, el Programa viene acompañando a los equipos docentes en la presentación y desarrollo de diferentes tipos de proyectos: Proyectos de Interés Social (PEIS); Proyectos de Extensión de Cátedra (PEC) y Proyectos de Extensión de Interés Institucional (PEII).

Los proyectos de extensión abordan una diversidad de temas, tales como: comercio solidario, consumo responsable; precio justo; promoción de huertas familiares; fortalecimiento de cooperativas de trabajo; autogestión, las vecinales; entre otros. El propósito de los proyectos es reflexionar en los ámbitos urbano y rural con los propios actores sobre la forma de construir una ESS y realizar acciones concretas para la promoción y fortalecimiento de las prácticas en el territorio.

Articulan espacios académicos, actores sociales y políticas públicas que potencian el trabajo de la Universidad en pos de mejorar la calidad de vida de la población.

De allí que una de las tareas centrales del Programa sea llevar a cabo un proceso de integración de los proyectos, estableciendo un funcionamiento sinérgico entre ellos y de las prácticas extensionistas existentes. Para ello se efectúan encuentros con el objetivo de socializar entre el conjunto de los proyectos las acciones realizadas, los resultados obtenidos, los problemas y obstáculos encontrados y las lógicas asociadas a su concepción de la ESS. De esta manera, se propicia el acompañamiento a las distintas acciones que emanan de los proyectos existentes, a fin de bregar por el trabajo en red.

Estos espacios de comunicación no quedan circunscriptos al ámbito académico. Hay espacios de socialización en el que participan los actores sociales involucrados en los proyectos, como también miembros de la Municipalidad y del Gobierno de la provincia de Santa Fe.

3.2. El Centro de Emprendedores de la Economía Social (CEES) Un ejemplo cabal de articulación entre el Estado y la Universidad se expresa en la figura del CEES.

En mayo de 2013, y a instancias del convenio firmado entre la UNL, el Ministerio de Desarrollo Social de la provincia de Santa Fe (a través de la Subsecretaría de Economía Social) y de la Municipalidad de la ciudad de Santa Fe (a través de la Oficina de Empleo), nace el CEES.

El Centro tiene el objetivo de generar un espacio de referencia que promueva, coopere y colabore con agentes, instituciones y 
organismos públicos y privados en el diseño y ejecución de procesos y estrategias de fortalecimiento de la ESS en la ciudad de Santa Fe. Entre sus propósitos, figuran:

- Brindar capacitación y asistencia técnica a través de cursos, talleres y jornadas de acuerdo a las necesidades y perfil de los emprendedores de la economía social.

- Constituir un espacio de encuentro e intercambio de experiencias entre emprendedores de la economía social.

- Promover la comercialización en el mercado local y regional.

- Vincular líneas de financiamiento para emprendedores y proyectos de la economía social.

- Diseñar mecanismos de evaluación que permitan medir impactos, resultados logrados, obstáculos, apropiación de tecnologías y conocimientos en el marco del proceso productivo.

- Propiciar el fortalecimiento de los emprendedores/as como grupo de acción colectiva, fomentando instancias de diálogo y debate que contribuyan a dar representatividad a sus intereses en el ámbito del Centro de Emprendedores.

EI PEESS aporta fundamentalmente a la formación y capacitación de los emprendedores y promueve la construcción de espacios asociativos.

\section{La docencia y la formación en ESS}

\subsection{Articulación con la cátedra de ESS}

En el año 2010 se creó la cátedra Economía Social y Solidaria ${ }^{1}$ con el propósito de presentar el debate en torno a la problemática de las iniciativas de economía social, su potencial transformador del actual sistema socioeconómico y el involucramiento interdisciplinar en su abordaje.

La cátedra se constituye en un espacio que estimula a la confrontación del análisis teórico a luz de la bibliografía obligatoria con las prácticas emergentes de casos seleccionados por el equipo de cátedra y por los estudiantes universitarios. De esta forma, se analizan diversas modalidades y experiencias de emprendimientos de ESS de la ciudad y se busca que los estudiantes interactúen fomentando el compromiso y aportando a la transformación social. En este sentido, con el transcurrir de los años de dictado, la cátedra se ha involucrado con el territorio, especialmente con las diferentes experiencias de ESS. Por mencionar sólo algunas de ellas, destacamos: la Cooperativa San Cayetano; la vecinal Villa del Parque; la Cooperativa Parque Bahía; la Cooperativa Setúbal; la

1) Se trata de una asignatura electiva para todas las carreras de la UNL (Res. C.S., 500/10) y optativa para las carreras de Contador Público Nacional y
Cooperativa TEKO y el Centro de Emprendedores de la Economía Social. Este trabajo territorial procura despertar en los estudiantes una sensibilidad hacia las experiencias de ESS a la vez que les permite operacionalizar el conocimiento brindado por la cátedra.

\subsection{Diplomado Virtual de ESS}

En 2014, en el marco del Programa ALFA III Universidades Estratégicas (de cooperación entre la Unión Europea y los países latinoamericanos), se coordinó y ejecutó juntamente con la Fundación Universitaria Católica del Norte y la UNL, el Diplomado Virtual "Perspectivas de la Economía Social y Solidaria; experiencias prácticas en el territorio".

El Diplomado profundizó aspectos referidos a la ESS a partir de la reflexión conjunta y de evidenciar avances teóricos y metodológicos entre docentes e investigadores de la UNL, la Universidad Nacional de General San Martín (Argentina); Universidade Estadual do Oeste do Paraná (Brasil); Florida Universitaria (España) y Católica del Norte (Colombia). El curso internacional contó con la participación de más de 50 alumnos de los diferentes países señalados entre los que participaron becarios del Programa ESS.

\subsection{Intercambio académico}

EI PEESS apuesta a la formación, actualización y capacitación de su equipo en instancias de intercambio académico a partir de la a firma de convenios internacionales con la Asociación de Universidades del Grupo Montevideo (AUGM), específicamente, con el Comité de Procesos Cooperativos e Iniciativas Económicas Asociativas (PROCOAS) y con el Programa de Movilidad para posgrados (ESCALA). Estos convenios estimulan el establecimiento de redes entre docentes, extensionistas e investigadores de la UNL con otras universidades nacionales o extranjeras vinculadas a la ESS. Un ejemplo de ello es el intercambio de docentes que se viene realizando en el marco del PROCOAS-AUGM, donde ya se han concretado más de 10 intercambios de docentes desde y hacia la UNL.

\subsection{Formación en temas de la ESS}

EI PEESS busca fomentar la formación de recursos humanos en el área de la ESS en el ámbito de la UNL. En la primera etapa, a modo de diagnóstico, efectuó un relevamiento de los recursos humanos de la Universidad vinculados a la temática. En esta línea, se ha invitado a participar en el Diplomado Virtual de la ESS, el cual fue cursado por estudiantes y docentes de nuestra Universidad. También el intercambio académico (comentado en el punto anterior) ha posibilitado a docentes cumplir instancias académicas en diferentes universidades de América Latina. Asimismo, en la cátedra de ESS se han llamado y realizado adscripciones de recientes graduados y estudiantes, a los cuales se les ha permitido luego incorporarse a los distintos proyectos de extensión e investigación atinentes al PEESS. 
Otra de las actividades fue la apertura al Programa del Voluntariado de la UNL, donde están participando más de 40 estudiantes voluntarios que se encuentran trabajando con las cooperativas de trabajo, en los proyectos de extensión y en el ámbito del CEES. El proceso de formación se ha efectuado hacia adentro y hacia afuera del ámbito universitario, y por ello también se llevó adelante un ciclo de conferencias titulado: "Hacia Otra Economía" durante el año 2014. En el marco del ciclo disertaron: Alejandro Rofman, Paula Rosa y Ariel García (UNGSM-CONICET); Susana Hintze (UNGS); Gerardo Sarachu Trigo (UdelaR, Uruguay); Cinthia Shammah (UBA-CONICET); Andrea Delfino (UNL-UNR) y Eliana Peretti (UNL); Esteban Cuatrín (UNL); Francesco Vigliariolo (UNSAM) y Rodolfo Pastore (UNQ).

\section{La investigación en ESS}

La investigación en el campo de la ESS presenta desafíos para el PEESS considerando su reciente creación. En el marco de Ciencia y Técnica de la UNL se trabaja particularmente con en el Programa de Investigación y Desarrollo Orientado a Problemas Sociales y Productivos, Ilamado CAID Orientado. Bajo esta línea se contemplan los siguientes proyectos:

"La Universidad Nacional del Litoral y las Cooperativas de Trabajo en Santa Fe: apoyo a su consolidación en el marco de la Economía Social y Solidaria" y "Mapeo, caracterización e interacción de los actores de la economía social y solidaria en los municipios de Santa Fe, Rosario y Reconquista", en desarrollo.

Además, en el contexto del Proyecto de Desarrollo Tecnológico y Social del Consejo Interuniversitario Nacional (PDTS-CIN) se aprobó el proyecto "Análisis de las iniciativas de economía social y solidaria en la ciudad de Santa Fe (2010-2014). Desarrollo de un mercado solidario".

\section{Consideraciones finales}

En este artículo hicimos un breve recorrido por el PEESS de la UNL, hicimos una presentación del mismo y de sus objetivos. Las actividades de extensión, docencia e investigación llevadas a cabo son instancias para seguir profundizando y evaluando para mejorarlas en pos de un acercamiento a la comunidad universitaria y extrauniversitaria.

La temática de la ESS, desde la institucionalización del Programa, se ha instaurado como una de las líneas a profundizar en la UNL como en otras universidades de Argentina. En este sentido, es un logro que en el corto plazo hayamos realizado numerosas actividades y presentado diferentes propuestas para el ámbito universitario. Otra oportunidad de intervención se lleva a cabo en el CEES, espacio de construcción de políticas entre diferentes escalas estatales, donde la Universidad —representada por el PEESS— representa un actor clave en la convergencia y divergencias de las propuestas que se motorizan y que tiene una acción directa sobre la comunidad santafesina.

De esta manera, consideramos acertado el camino que hemos definido en la manera de llevar a cabo la vinculación con otros actores por medio de la extensión. El desafío se presenta en la investigación en la ESS al tratarse de una temática relativamente nueva para la Universidad.

Por lo tanto, en el presente artículo quedan planteados los logros, límites y desafíos de gestión que se presentan en el PEESS para construir la ESS desde la Universidad, tarea en constante construcción y diálogo con los diferentes actores universitarios y extrauniversitarios.

\section{Referencias bibliográficas}

Arroyo, D. (2008). La Economía social como estrategia de inclusión. Diplomatura en Economía Social y Desarrollo Local. Buenos Aires: FLACSO

Bowles, S. y Edwards, R. (1985). Introducción a la economía: competencia, autoritarismo y cambio en las economías capitalistas. Madrid: Alianza Universidad.

Coraggio, J.L. (2005). ¿Es posible otra economía sin (otra) política? En Colección El Pequeño Libro Socialista. Buenos Aires: Editora La Vanguardia.

Coraggio, J.L (2007). Economía social, acción pública y política (hay vida después del neoliberalismo). Buenos Aires: CICCUS.

Da Cruz, A.C.M. (2006). A Diferenca da Igualdad. A dinámica da economía

solidária em quatro ciudades do Mercosul. SP, Brasil: Universidade Estadual do Campinas.

Lafferriere, L.; Tealdo J.C.y Azerrad, R. (2010). Construyendo redes de Economía Social entre la Universidad, Organizaciones Sociales y el Gobierno Local. Documento de Trabajo. PEII- Proyecto de Extensión de Interés Institucional. Universidad Nacional del Litoral, Santa Fe.
Nosetto, L. (2005). La vigencia de la Economía Social en tanto sintonía del sustrato social del trabajo humano. Ponencia presentada en el $7^{\circ}$ Congreso Nacional de Estudios del Trabajo. Buenos Aires.

Polanyi, K. (2011). La Gran transformación. Los orígenes políticos y económicos de nuestro tiempo. $1^{\circ}$ reimpresión. Buenos Aires: Fondo de Cultura Económica. Rapoport, M. y Brenta, N. (2010). Las grandes crisis del capitalismo contemporáneo. Buenos Aires: Capital Intelectual.

Razeto, L. (1997). Los caminos de la economía de solidaridad. Buenos Aires: Lumen-Hvmanitas.

Singer, P. (2000). Economía solidária: um modo de produção e de distribuição. En Singer, P.; Souza, A.R. et al., Economia solidária no Brasil: a autogestão como resposta ao desemprego. São Paulo: Contexto.

Tealdo, J.C. (2014). El programa de extensión economía social y solidaria en la Universidad Nacional del Litoral. Ponencia presentada III Jornadas de Extensión del Mercosur. Tandil. 